\title{
Réhabilitation implanto-prothétique fixe d'un maxillaire par implantation post-extractionnelle et mise en charge immédiate
}

\section{RÉSUMÉ}

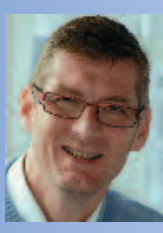

Christophe DESCHAUMES

CHU de Clermont-Ferrand,

Service d'Odontologie.

Université d'Auvergne Clermond-Ferrand I

Faculté de Chirurgie Dentaire.

Inserm, U929.

11, boulevard Charles-de-Gaulle,

63000 Clermont-Ferrand, France.

Julien DUROUX

Faculté de Chirurgie Dentaire et Service d'Odontologie

de Clermont-Ferrand

EA 3847.

Didier COMPAGNON

Faculté de Chirurgie Dentaire et Service d'Odontologie de Clermont-Ferrand.

Laboratoire de recherche clinique en prothèse odontologique.

Cédric HUARD

Faculté de Chirurgie Dentaire et Service d'Odontologie de Clermont-Ferrand.

Emmanuel NICOLAS

Faculté de Chirurgie Dentaire et Service d'Odontologie de Clermont-Ferrand.

EA 3847.

Sylvain OPÉ

Faculté de Chirurgie Dentaire et Service d'Odontologie de Clermont-Ferrand.

EA 3847.

Manuel VASCONCELOS

Faculté de Chirurgie Dentaire et Service d'Odontologie de Clermont-Ferrand.

Laboratoire de prothèse dentaire ARV.

Pascal AUROY

Faculté de Chirurgie Dentaire et Service d'Odontologie de Clermont-Ferrand.

Laboratoire de recherche clinique en prothèse odontologique.

Jean-Luc VEYRUNE

Faculté de Chirurgie Dentaire et Service d'Odontologie

de Clermont-Ferrand.

EA 3847.

Maurice MORENAS

Faculté de Chirurgie Dentaire et Service d'Odontologie

de Clermont-Ferrand.

Laboratoire de recherche clinique en prothèse odontologique.

Le concept de mise en charge immédiate et celui de l'implantation post-extractionnelle immédiate ont tous les deux connu un engouement ces quinze dernières années et de nombreuses études cliniques ont validé ces deux protocoles. Depuis une dizaine d'années, quelques équipes ont associé les deux concepts pour développer l'implantation post-extractionnelle avec mise en charge immédiate. Outre la rapidité du traitement, l'absence de port de prothèse provisoire adjointe mal tolérée et le retour rapide de la fonction de mastication, ce protocole facilite la cicatrisation tant au niveau osseux que muqueux. Le but de cet article est de montrer les intérêts et les contraintes de cette technique et de présenter un protocole pour une réhabilitation complète d'un maxillaire.

- implants dentaires

- post-extractionnel

- mise en charge immédiate
AOS 2011;256:289-304 DOI: $10.1051 /$ aos/2011403

(C) EDP Sciences 


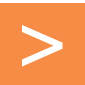

Ces vingt dernières années ont vu une évolution importante dans le domaine de l'implantologie. Les principes de Brånemark prônant la pose de l'implant sur un os cicatrisé, son enfouissement pour en obtenir l'ostéointégration avant de le mettre en charge, ont été revus voire balayés. Dès les années 1990, l'implantation immédiatement après I'avulsion dentaire et la mise en charge immédiate après la pose des implants sont deux procédés qui ont été simultanément développés pour réduire, notamment, le temps de traitement. Si ces techniques pouvaient être considérées comme empiriques au début, de nombreuses études les ont depuis validées. Ces dernières années, une autre étape a été franchie en asso- ciant l'implantation post-extractionnelle et la mise en charge immédiate. L'intérêt d'une telle technique réside dans la réduction du nombre de séances, l'inutilité du port d'une prothèse amovible transitoire mal vécue par le patient, le maintien voire l'augmentation des volumes des tissus osseux et muqueux péri-implantaires, une fonction masticatoire et une esthétique retrouvée immédiatement après les avulsions. L'objectif de cet article est de montrer que cette technique est valide et réalisable avec des procédures accessibles à tout praticien implantologiste. Nous reprendrons point par point les principes qui ont conduit à valider l'implantation post-extractionnelle avec mise en charge immédiate.

\section{Implantation post-extractionnelle immédiate}

Depuis la première publication [1], cette technique a été très régulièrement publiée et validée. Cependant, il est difficile de définir les règles de mise en place de ces implants tant elle dépend de l'état du site d'implantation, du profil et de la surface implantaire ainsi que des techniques de régénération osseuse pouvant être associées.

Il convient de distinguer l'implantation retardée, où l'implant est posé après cicatrisation muqueuse de l'alvéole, de l'implantation immédiate qui a lieu consécutivement à l'avulsion de la dent [2].

\section{Quels sont les intérêts} et les contraintes de l'implantation post-extractionnelle?

Les avantages sont essentiellement la préservation du capital osseux, sachant que les deux tiers de la résorption osseuse totale a lieu dans les trois premiers mois après l'avulsion dentaire. Schropp et al. [3] montrent le fort potentiel cicatriciel ostéogénique au niveau du site d'avulsion: la régénération osseuse est plus importante si les implants sont placés immédiatement que s'ils sont mis en place trois mois après l'avulsion, cela en l'absence de perte de paroi alvéolaire.

La mise en place de l'implant facilite donc la conservation des procès alvéolaires et notamment les septa interdentaires et donc aide au maintien des papilles qui dépend de l'os sousjacent. Elle permet alors une meilleure intégration de l'implant au niveau esthétique et la conservation d'un rapport couronne clinique / implant plus satisfaisant.

Dans les cas de maladie parodontale avec défaut osseux important, une régénération osseuse guidée peut être réalisée en même 
temps que la mise en place de l'implant et permettre un gain osseux à long terme [4].

Les autres avantages de ce concept sont la réduction du temps de traitement et du nombre d'interventions chirurgicales, d'où une diminution des coûts pour les actes chirurgicaux, avec en parallèle, une meilleure acceptation du traitement par le patient.

Les difficultés de l'implantation immédiate sont essentiellement d'ordre technique. La première concerne le manque d'os résiduel à l'apex de I'alvéole qui peut compromettre la stabilité de l'implant. La seconde réside dans le fait que l'implant ne doit pas être placé au milieu de l'alvéole et dans l'axe de celle-ci mais au dépend de ses parois pour assurer son ancrage. Une régénération osseuse guidée peut être entreprise si le hiatus laissé entre l'implant et la paroi alvéolaire est supérieur à $2 \mathrm{~mm}$. La troisième est liée au manque de gencive attachée ou à la présence d'un biotype gingival fin qui rendra difficile le sertissage gingival de l'implant. Des lambeaux déplacés coronairement ou latéralement peuvent régler ce problème mais compliquent la chirurgie et peuvent compromettre plus ou moins le résultat final.

En présence des situations cliniques sus-décrites, la procédure d'implantation post-extractionnelle peut s'avérer délicate en fonction du site et s'adresse alors à des praticiens expérimentés.

\section{Quels sont les facteurs de succès de l'implantation post-extractionnelle?}

Ils sont liés à la technique d'avulsion qui doit être le plus atraumatique possible, afin de conserver les parois osseuses, notamment la paroi vestibulaire. Le curetage et le lavage de I'alvéole doivent éliminer tout débris dentaire, osseux et les tissus de granulation qui peuvent être source soit d'une infection périimplantaire, soit d'une fibro-intégration de l'implant.

Ils sont également liés à l'implant. Celui-ci doit être fileté et avoir un état de surface rugueux (sablé / mordancé ou traité par un spray de plasma de titane) pour en augmenter la surface de contact avec l'os, donc sa stabilité primaire. Le profil de l'implant donne lieu à beaucoup de débats : certains préfèrent l'implant conique ou cylindro-conique pour mieux s'appuyer sur les parois de l'alvéole et avoir une meilleure stabilité ; d'autres préfèrent l'implant cylindrique pour conserver un hiatus entre l'implant et la paroi vestibulaire et favoriser la cicatrisation osseuse par comblement de celui-ci sans résorption non contrôlée de la corticale vestibulaire. Cette stabilité du procès alvéolaire est indispensable à la stabilité de la gencive attachée vestibulaire. D'autres auteurs prônent l'intérêt du switching platform ou du festonnage du col implantaire [5].

\section{Cette technique est-elle fiable?}

Cette fiabilité a été largement décrite dans la littérature. Les taux de succès à 5 ans sont compris entre $92 \%$ et $94,7 \%[6,7]$.

Reprenons maintenant les principes de la deuxième partie du concept de la technique d'implantation post-extractionnelle avec mise en charge immédiate. 


\section{Mise en charge immédiate}

De nombreuses études ont montré le caractère empirique du principe de Branemark qui imposait une période de cicatrisation sans contrainte de 4 à 6 mois. La technique de mise en charge immédiate, empirique à ses débuts, a été aujourd'hui largement validée.

Elle est définie par la pose d'une prothèse fonctionnelle, en occlusion avec les dents antagonistes dans les 48 heures suivant l'implantation. On parle de mise en charge précoce si la prothèse est placée entre $48 \mathrm{~h}$ post-implantation et 3 mois post-implantation et de mise en charge conventionnelle après une période d'ostéointégration de 3 mois [8].

\section{Quels sont les intérêts}

et les contraintes

de la mise en charge

\section{immédiate?}

Le principal intérêt en cas d'implantation multiple est la solidarisation des implants entre eux ; ceci est d'autant plus vrai si la prothèse implanto-portée remplace plusieurs secteurs et donc stabilise les implants de façon tridimensionnelle. La mise en charge immédiate évite ainsi la sollicitation des implants isolés, par une prothèse amovible transitoire. Du point de vue osseux, la mise en charge immédiate apparait permettre un meilleur maintien de I'os péri implantaire à long terme par rapport à la mise en charge conventionnelle. Cependant, que la mise en charge soit immédiate ou différée à 7 jours ou à 14 jours, la réponse osseuse périimplantaire est la même : le pourcentage de contact os / implant, la densité osseuse et la perte osseuse péri-implantaire ne varient pas d'une technique à l'autre [9].
On pourrait légitimement penser que la pose d'une prothèse immédiate implanto-portée fixée favorise l'intégration gingivale péri-implantaire. Cependant, il semble qu'il n'y ait pas de différence entre une mise en charge immédiate et une mise en charge différée, concernant l'attache gingivale péri-implantaire (hauteurs de l'épithélium de jonction, du tissu de conjonction et de l'espace biologique, épaisseur de la gencive kératinisée) [10,11]. En revanche, le maintien des papilles, donc l'esthétique, est meilleur lors d'une mise en charge immédiate que lors d'une mise en charge conventionnelle [11].

Mais, du point de vue du patient, l'intérêt réside dans la réduction drastique du nombre de séances : deux séances suffisent pour le traitement exclusivement implantaire (hors celles de contrôle): I'une pour la pose des implants, l'autre pour la pose de la prothèse. De plus, le patient évite le port d'une prothèse amovible plus ou moins stable du fait du dégagement de I'intrados de celle-ci au niveau des implants afin de ne pas les solliciter. L'acceptabilité du traitement est donc grandement améliorée par cette technique qui permet de retrouver les fonctions masticatrice, phonatoire et une esthétique dans les jours qui suivent l'implantation [12].

Toutefois, la mise en charge immédiate nécessite un plus grand nombre d'implants. Pour la réhabilitation d'une arcade complète, il est préférable de poser 5 à 6 implants à la mandibule [13] et 6 à 8 implants au maxillaire. Néanmoins, il faut pondérer ces chiffres en rappelant des taux de succès à long terme identiques à la mandibule avec seulement 4 implants $(98,1 \%$ à 5 ans et $94,8 \%$ à 10 ans) $[14,15]$. On observerait les mêmes taux de succès au maxillaire avec 4 ou 6 implants [16]. 
Enfin, il faut ajouter que les patients ayant bénéficié de ce type de protocole, ont une difficulté à maintenir une hygiène rigoureuse, notamment les premières semaines [17], ce qui impose des visites de contrôle régulières.

\section{Cette technique est-elle fiable?}

La fiabilité de cette technique a été décrite dans la littérature. Cependant comme les situations cliniques sont nombreuses, il est difficile de statuer pour toutes. Concernant la réhabilitation fixe d'une arcade entière, les publications sont abondantes pour la mandibule et montrent des taux de succès éloquents de 98 à $100 \%$ après 3 et 6 ans post-implantation, selon les études $[18,19]$.

Au maxillaire, les études sont moins nombreuses; le taux de succès varie de $97 \%$ à $99 \%$ entre 2 et 3 ans [20], sans différence significative avec la mandibule [18].

Il semble en fait qu'il n'y ait aucune différence significative de taux de succès entre une mise en charge conventionnelle $(95,9 \%)$ et la mise en charge immédiate $(93,9 \%)$ à 3 ans [21]. Des résultats similaires sont retrouvés dans d'autres études $[4,22,23]$.

\section{Quels sont les facteurs de succès de la mise en charge immédiate?}

Ils sont tout d'abord liés à la chirurgie. La stabilité de l'implant est le facteur principal de succès d'une mise en charge immédiate surtout dans les cas de restauration unitaire ou d'un secteur de petite étendue. Il est nécessaire, dans ces configuration cliniques, d'avoir une stabilité primaire obtenue par un vissage de l'implant à $35 \mathrm{Ncm}$. Si tel n'est pas le cas, une mise en charge conventionnelle est préférable [8]. Dans le cadre d'une reconstruction de l'arcade complète avec 6 ou 8 implants, si le manque de stabilité d'un implant n'est pas rédhibitoire, le risque d'échec augmente si plusieurs implants n'ont pas de stabilité primaire. Les implants devront également être répartis le plus uniformément pour éviter notamment les extensions distales trop importantes et les pontiques de grande étendue.

Ils sont également liés à la prothèse : la prothèse immédiate fixe doit être rigide pour solidariser au maximum les implants entre eux, donc doit posséder une infrastructure métallique. Le réglage de l'occlusion de celle-ci doit répartir les contacts occlusaux sur toute l'arcade et éviter sinon répartir au maximum les forces horizontales ou obliques, déstabilisatrices pour les implants, lors des mouvements de latéralité et / ou de propulsion. Les parafonctions et le bruxisme sont des facteurs de risque qui, soit contre-indiquent la mise en charge immédiate, soit imposent d'augmenter le nombre d'implants. Mais, le traitement préalable ou conjoint de ces parafonctions est indispensable.

La mise en charge immédiate nécessite-t-elle une planification par ordinateur avec réalisation d'un guide chirurgical issu de la numérisation?

De nombreux systèmes de planification sur scanner ont été développés; les plus utilisés sont Nobelguide ${ }^{\circledR}$, Simplant ${ }^{\circledR}$ et Easyguide ${ }^{\circledR}$. Ils permettent la réalisation d'un guide chirurgical d'une grande précision après la planification informatique de la position et de l'axe des implants sur des images scanner [24]. La justifi- 
cation de cette technique est d'ordre chirurgical et prothétique.

D'un point de vue chirurgical, la planification par l'analyse sur le scanner permet l'optimisation des sites osseux résiduels et évite souvent les greffes d'apposition et les comblements sinusiens $[25,26]$. Cet argument peut être contré. En effet, les implants courts (moins de 8 millimètres de longueur) ont un même taux de fiabilité que ceux plus longs et permettent souvent d'éviter ces techniques d'apposition. Mais la planification permet également de placer et d'orienter les implants le plus favorablement par rapport aux axes des dents prothétiques. Elle autorise un acte chirurgical plus rapide puisque le chirurgien est guidé lors du forage, sans possibilité de modification de l'axe. Son grand avantage réside dans l'absence de nécessité de lambeau mucopériosté pour poser les implants [27]. Les suites postopératoires sont alors plus simples et l'intégration gingivale des implants, meilleure. Cependant, il faut noter que l'implantation sans lambeau peut être souvent réalisée, même sans I'aide de ces guides chirurgicaux issus de la planification.

En revanche, un des risques de cette technique est l'instabilité ou le mauvais positionnement du guide chirurgical, ce qui faussera irrémédiablement les sites d'implantation et les axes des implants, d'où des échecs spectaculaires ! Une étude de Abad-Gallegos rapporte un taux d'échec par implant de 8,2 \% , incriminant souvent le manque de stabilité des implants [28].

Du point de vue prothétique, la planification permet la réalisation préopératoire d'une prothèse immédiate fixe avec une armature rigide qui s'adaptera de façon passive, si les implants sont placés dans la position voulue ! La moindre erreur lors du temps chirurgical fera perdre à la prothèse sa passivité [28]. Le gain de temps se retrouve également à cette étape. Du point de vue du patient, ces techniques ont un coût encore important et peuvent donc être dissuasives.

Mais, lorsqu'on veut se passer de cette technique, il est alors indispensable d'avoir un laboratoire de prothèse réactif et expérimenté, à proximité du cabinet, qui soit capable de réaliser une prothèse à armature métallique en 48 heures.

Il faut conclure en rappelant que ces techniques assistées par ordinateur, présentées initialement comme simples d'utilisation, ne doivent pas être mises entre toutes les mains, mais doivent être réservées aux chirurgiens expérimentés.

\section{Est-ce que les techniques} de préparation osseuse peuvent compromettre la mise en charge immédiate?

La condensation osseuse par ostéotome, utilisée au maxillaire, permet une augmentation de la densité osseuse et du pourcentage de contact osseux à la surface implantaire par rapport une technique classique de forage. Elle favorise donc la mise en charge immédiate [29].

De même, la mise en place d'implants mis en charge immédiatement dans une greffe osseuse d'apposition ou une élévation du plancher sinusien n'altère pas les taux de succès implantaires $[30,31]$.

La régénération osseuse guidée lors de la pose d'implant mis en charge immédiatement ne modifie pas non plus le taux de succès et n'influence ni l'évolution du niveau osseux, ni celle du contour gingival [32]. 


\section{Mise en charge immédiate avec implantation post-extractionnelle}

Ainsi que nous l'avons décrit, les techniques d'implantation post-extractionnelle immédiate et celles de mise en charge immédiate ont connu un fort engouement ces dernières années et été validées comme fiables. Forts des constatations préalablement énoncées, c'est tout naturellement que certains ont associé ces deux concepts.

\section{Cette technique est-elle fiable?}

Le taux de succès varie entre $94,2 \%$ et $100 \%$ selon le site implanté, selon la situation et l'étendue de l'édentement (unitaire, partielle ou complète) et la nature de la reconstruction prothétique implanto-portée (adjointe, fixe) $[4,10,23,33-38]$.

Nous considèrerons ici sur la reconstruction implanto-portée fixe complète.

\section{Quels sont les intérêts} et les limites

de la reconstruction complète d'un maxillaire par implantation post-extractionnelle et mise en charge immédiate?

Les intérêts sont ceux préalablement énoncés supra pour l'implantation post-extractionnelle conjugués à ceux pour la mise en charge immédiate. L'intérêt principal est surtout l'acceptabilité du traitement par le patient: au maximum 48 heures après les avulsions de ses dents et la pose des implants, le patient retrouve les fonctions masticatrice, phonatoire et... son sourire.
Le nombre de séances, même si celles-ci sont longues est réduit au nombre de deux, hors séances de contrôle ultérieures, pour tout le traitement.

Les limites sont parallèlement celles de l'implantation post-extractionnelle et celles de la mise en charge immédiate en sachant que le cumul de difficultés non ou mal gérées conduit facilement à la perte d'implants ou à l'impossibilité de poser la prothèse immédiate.

Est-ce que la pose d'implants à la place de dents présentant une parodontite compromet l'implantation post-extractionnelle avec mise en charge immédiate?

Les patients candidats à cette technique ont souvent un long passé dentaire et présentent des parodontites terminales. Perdre leurs dents restantes et se retrouver avec une prothèse amovible, même si elle est transitoire, leur est difficile à accepter et il est pertinent de leur proposer cette technique. Différentes études cliniques sur des patients ayant une parodontite ont montré que le taux de succès, le gain d'os péri-implantaire et l'index papillaire sont les mêmes que les implants post-extractionnels soient mis en charge immédiatement ou qu'ils le soient après ostéointégration [32,39].

Cependant, l'état parodontal au niveau des implants post-extractionnels influence tout de même la cicatrisation muqueuse péri-implan- 
taire. Crespi a montré que le risque de récession gingivale péri-implantaire est plus important si la hauteur de gencive kératinisée au niveau de la dent est inférieure à $2 \mathrm{~mm}$. Cette différence se retrouve pour les autres indices parodontaux (indice de plaque modifié, indice de saignement, indice gingival) [40]. Un aménagement tissulaire par greffe conjonctive enfouie, par exemple, doit alors être prévu lors de l'implantation dans une telle situation parodontale préexistante.

\section{Technique de mise en charge immédiate post-extractionnelle dans le cadre d'une réhabilitation complète}

Nous développerons ici un protocole de mise en charge immédiate avec implantation postextractionnelle sans guide chirurgical issu d'une planification informatique que nous utilisons régulièrement.

\section{Cas clinique}

M. C. présente une édentation prémolo-molaire maxillaire bilatérale suite à une parodontite de I'adulte. II ne subsiste au maxillaire que le groupe incisivo-canin. Ces dents ont régulièrement des abcès parodontaux. A la mandibule, il $y$ a également des poches parodontales stabilisées par la maintenance parodontale. L'indication d'avulsion des dents restantes maxillaires avec pose $d^{\prime}$ implants post-extractionnels et mise en charge immédiate d'un bridge implanto-porté est posée.

\section{$>$ Étude préopératoire}

L'examen clinique montre des crêtes larges au niveau des secteurs édentés et une résorption verticale. Un montage prospectif est réalisé avec évaluation du futur schéma occlusal. Les incisives centrales et canines sont considérées en position correcte. L'orthopantomogramme montre une faible hauteur d'os disponible en regard des sinus maxillaires (fig. 1). Un scanner est inutile dans ce cas, car le volume osseux dans le plan frontal a été considéré cliniquement comme suffisant. A partir du montage prospectif, un guide chirurgical est confectionné à partir d'une plaque thermoformée rigide. Ce guide servira pour le repérage des sites de forage au niveau des secteurs édentés et pour le choix de l'axe des implants qui doit être au plus près de l'axe prothétique. II recouvre les dents restantes et préfigure les dents prothétiques dans les secteurs édentés (fig. 2). Pour avoir une répartition harmonieuse des implants, les sites 17,15, 13, 11, 21, 23, 25 et 27 sont retenus. Un porte empreinte individuel est également réalisé par le laboratoire. Un duplicata du montage prothétique, ajouré au niveau des sites d'implantation prémolomolaires, va servir de maquette d'occlusion.

\section{> Étape chirurgicale}

L'intervention est réalisée sous anesthésie locale. Le repérage des implants au niveau des secteurs édentés est fait par forage transmuqueux avec le guide chirurgical en place (fig. 3). 
Les dents restantes sont avulsées et les alvéoles curetées pour éliminer les tissus de granulation. Le forage des implants incisivo-canins est fait avec le guide chirurgical repositionné pour obtenir une émergence et un axe des implants compatible avec la future prothèse (fig. 4 et 5 ). Les implants sont posés sur ces sites. La gencive est incisée avec un bistouri circulaire (punch) au niveau des sites des implants prémolaires et molaires et l'os mis à nu est cureté (fig. 6). La hauteur osseuse disponible étant légèrement insuffisante au niveau des prémolaires, une élé-

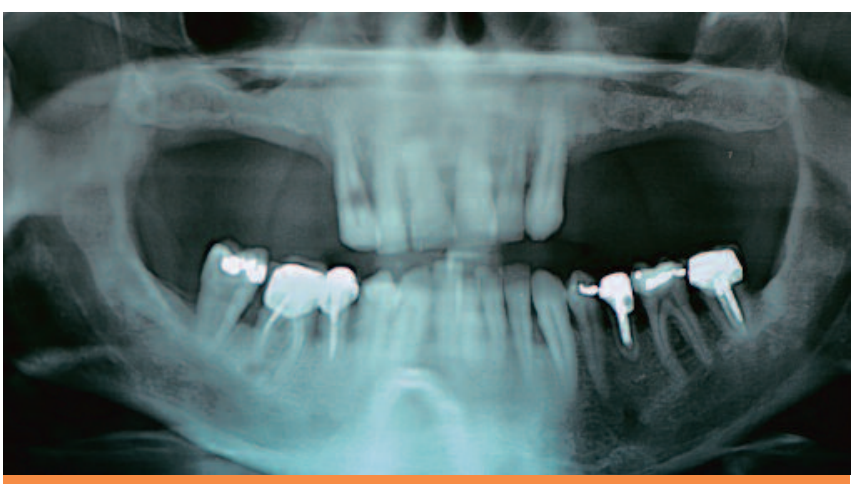

Fig: 1 Orthopantomogramme préopératoire.

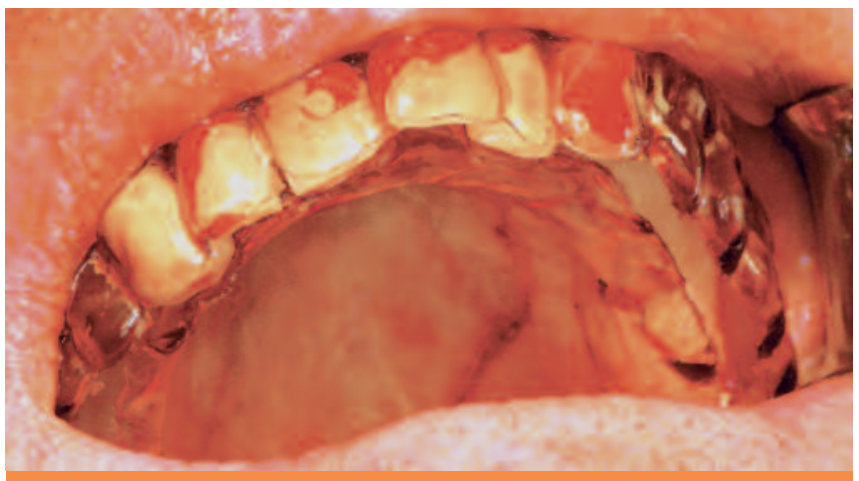

Fig. 2 Guide chirurgical en place.

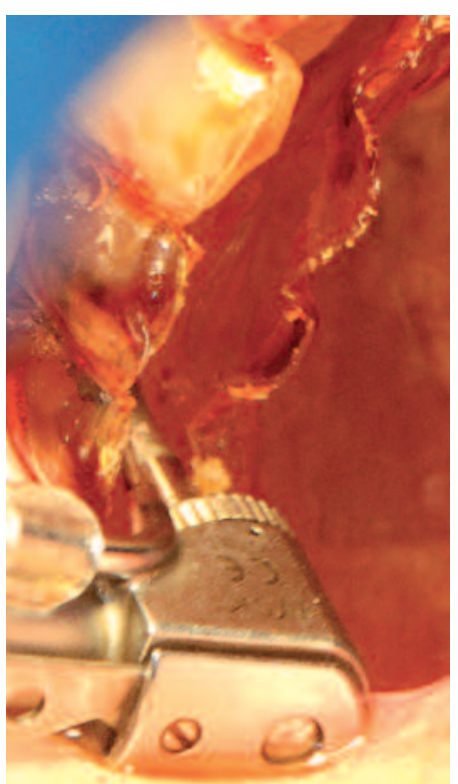

Fig. 3 Indexation de la muqueuse au niveau des sites implantaires des zones édentés.

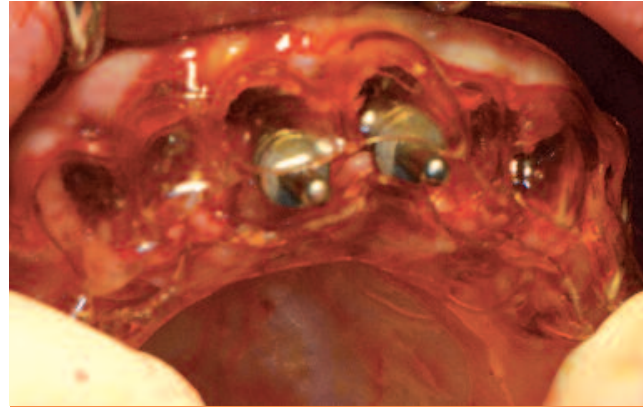

Fig. 4 Axe de forage initial au niveau des incisives selon le guide chirurgical.

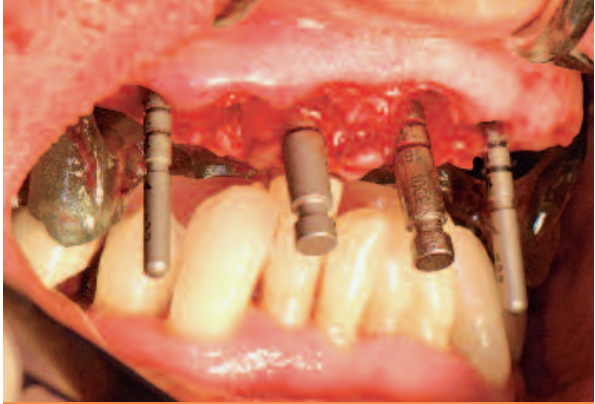

Fig. 5 Axe de forage des implants au niveau des incisives et canines.

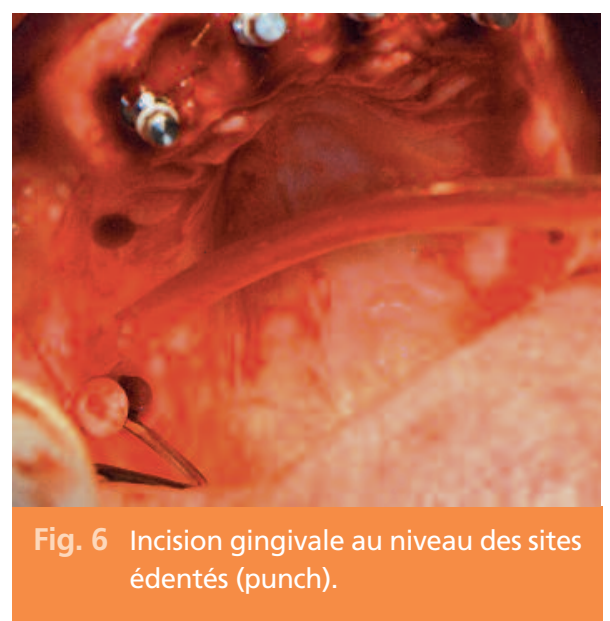


vation du plancher sinusien est faite par ostéotome selon la technique de Summers (fig. 7). La préparation implantaire est faite par forage au niveau des sites molaires. Les implants sont posés. Des transferts d'empreinte sont mis en place (fig. 8) et les sutures sont réalisées.

\section{> Prise d'empreinte}

On vérifie que le porte-empreinte individuel s'insère sans interférence avec les transferts (fig. 9) et l'empreinte est réalisée avec un polyéther (fig. 10).

\section{$>$ Enregistrement des relations intermaxillaires}

Des butées d'occlusion sont placées sur les implants prémolaires et incisifs et ajustées pour éviter des interférences avec la maquette d'occlusion (duplicata du montage prospectif) et avec les dents antagonistes (fig. 11).

Les butées prémolaires sont solidarisées à la maquette d'occlusion avec de la résine autopolymérisante (fig. 12). Les relations intermaxillaires sont enregistrées avec une silicone d'occlusion qui solidarise par la même occasion les butées incisives à la maquette (fig. 13). Ces butées solidarisées au duplicata du montage par la silicone permettent un repositionnement fiable de celui-ci sur les analogues d'implants du modèle de travail.

Le patient est alors transféré en salle de repos puis un orthopantomogramme de contrôle est réalisé (fig. 14).

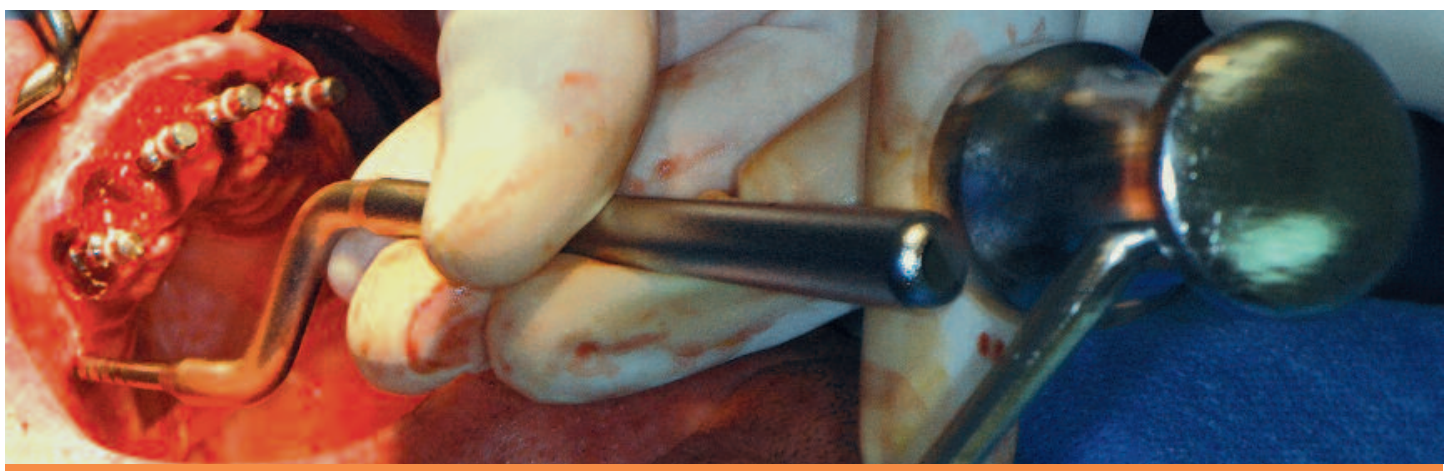

Fig. 7 Mise en forme des sites implantaires prémolaire par ostéotome (technique de Summers).
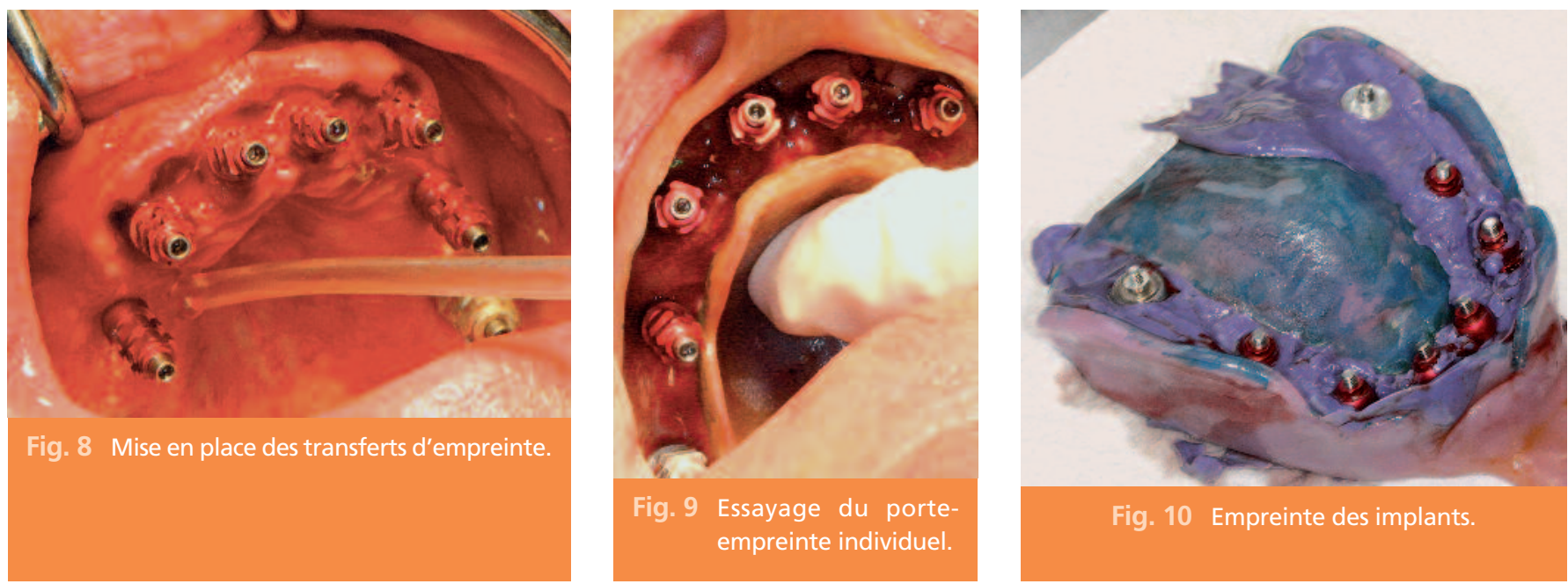
$>$ Pose de la prothèse

Quarante-huit heures après, le bridge est posé

(fig. 15). Le couple de serrage des piliers prothé- tiques dépend de la stabilité initiale des implants.

Il doit impérativement être inférieur à celui des implants sous peine de déstabiliser ceux-ci.

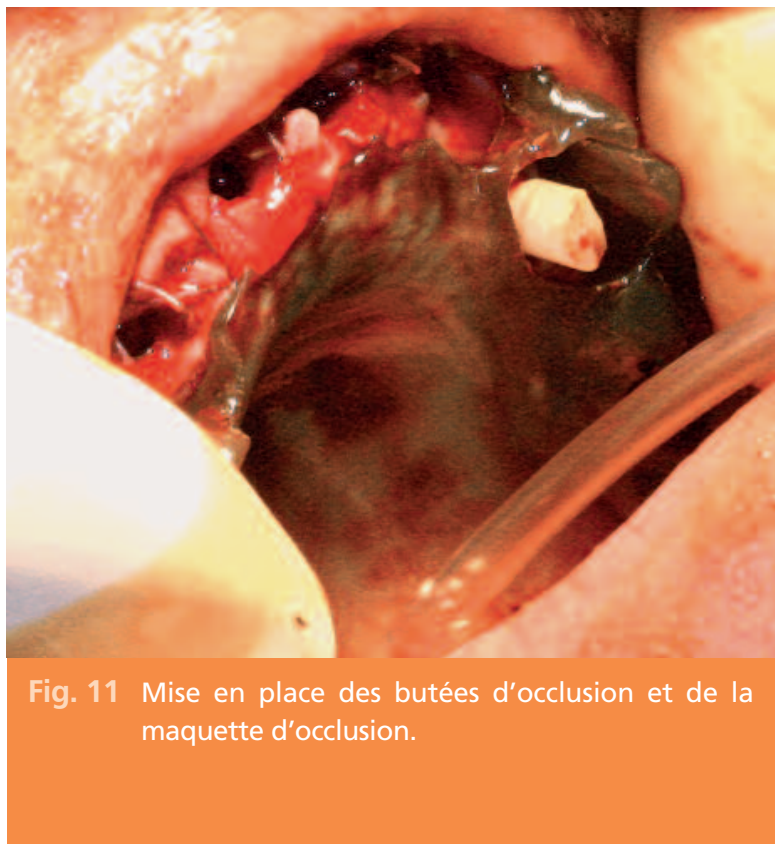

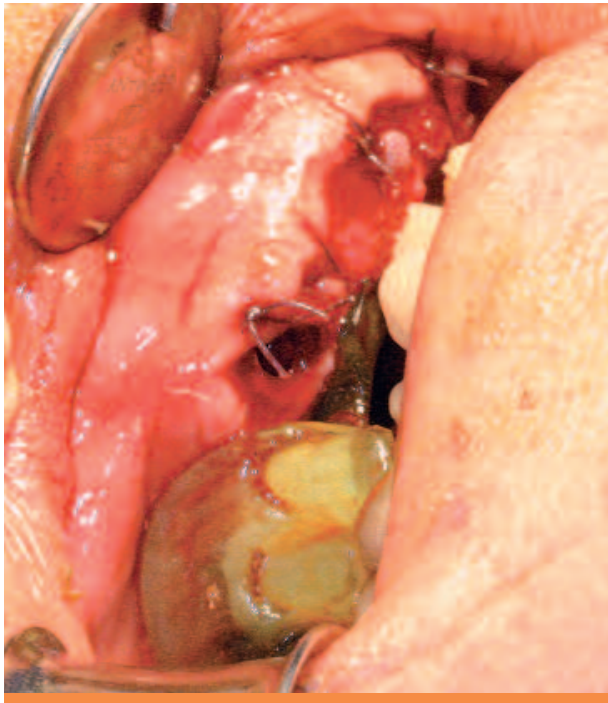

Fig. 12 Solidarisation des butées d'occlusion prémolaires avec la maquette d'occlusion.

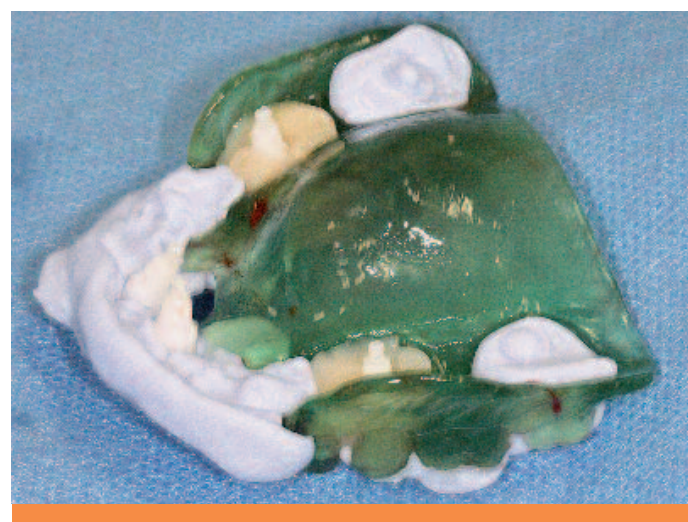

Fig. 13 Enregistrement des relations intermaxillaires.

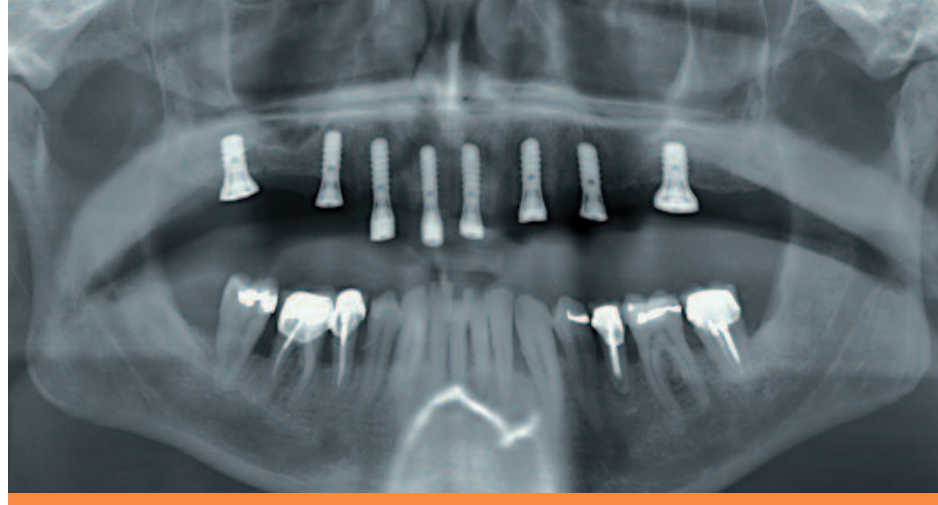

Fig. 14 Orthopantomogramme après implantation.

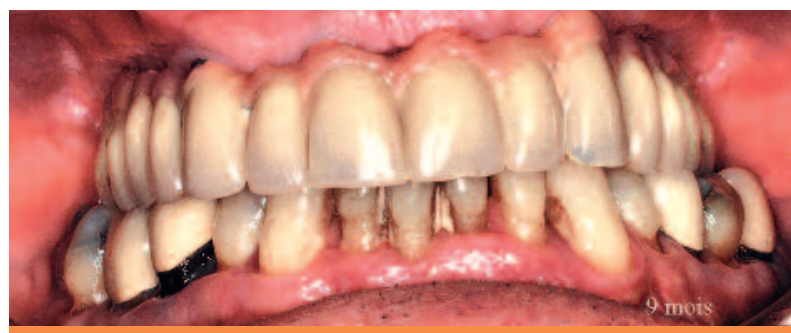

Fig. 15 Bridge provisoire mis en place (vue à 9 mois). 
La passivité de l'armature est contrôlée par des clichés radiographiques. Un réglage de I'occlusion est fait en bouche de façon à répartir harmonieusement les contacts occlusaux et à éviter les contacts non travaillants dans les mouvements de latéralité. Une fonction de groupe est recherchée.

\section{Discussion}

La réalisation de modèles d'étude et un montage prospectif avec évaluation du futur schéma occlusal sont des éléments indispensables pour valider le futur projet prothétique. Cette étape est importante puisque c'est à partir de ce montage que seront réalisés le guide chirurgical, le porte-empreinte individuel, la maquette d'occlusion et la correction occlusale ou les reconstructions prothétiques de l'arcade antagoniste.

L'examen tomodensitométrique, avec guide radiologique issu du montage prospectif, est seulement indiqué en cas d'incertitude après l'examen clinique et l'étude de l'orthopantomogramme ou des radiographies rétro-alvéolaires et en cas d'atrophie osseuse sévère.

Concernant le guide chirurgical, ce peut être soit une plaque thermoformée, si la position et l'axe des dents prothétiques correspondent à celles du patient, soit un duplicata en résine transparente du montage prospectif, s'il y a des édentements préexistants ou si la position et l'axe des dents naturelles ne sont pas conservés. Le guide radiologique peut également être utilisé comme guide chirurgical.

La phase chirurgicale peut être réalisée sous anesthésie générale, mais les conditions d'installation du patient et l'anesthésie par elle-même rendent plus difficile la pose des implants, l'empreinte mais surtout l'enregistrement de la relation intermaxillaire. Aussi, I'anesthésie locale
Le patient est revu les semaines suivantes pour contrôle et réglage de l'occlusion, contrôle de I'hygiène et dépose de points de suture à 15 jours. La prothèse d'usage ne sera réalisée qu'après un an. Si nécessaire, les profils d'émergence seront modifiés entre temps pour guider la cicatrisation muqueuse.

avec une analgésie par perfusion de paracétamol pendant l'intervention est préférable. Cependant le délai disponible d'intervention ne peut excéder 2 heures, au-delà le patient devient moins coopérant.

L'enregistrement de la relation intermaxillaire peut être réalisé de différentes façons.

Soit on avulse les dents et on pose simultanément des implants dans un premier secteur. La relation intermaxillaire est alors enregistrée au niveau de ce secteur avec une silicone d'occlusion, après avoir positionné des butées occlusales dans les implants. Les avulsions et la pose des implants sont ensuite réalisées en controlatéral et la relation intermaxillaire de ce $2^{\mathrm{e}}$ secteur est enregistrée de la même façon après avoir repositionné le silicone et les butées du premier secteur.

Si le montage prospectif ne correspondait pas à la position et à l'axe des dents naturelles, toutes les dents sont avulsées dans un premier temps en ne conservant que 3 ou 4 dents réparties sur les deux hémi-arcades, ceci afin de conserver les relations intermaxillaires. Les premiers implants sont posés et la relation intermaxillaire est enregistrée avec des butées d'occlusion sur ceux-ci. L'avulsion des dents restantes et la pose des derniers implants sont ensuite réalisées [41].

En cas d'édentements postérieurs, l'utilisation d'une maquette d'occlusion stabilisée sur les implants est indispensable (cf. cas clinique). 
Concernant le type de piliers prothétiques, nous optons habituellement pour une réalisation de la prothèse transitoire sur piliers définitifs et non sur piliers transitoires. En effet, le changement de pilier lors de la réalisation de la prothèse d'usage provoque une perte osseuse significativement plus importante que si les piliers définitifs sont mis en place immédiatement après la pose des implants [42].

Concernant l'occlusion, le choix entre celle en relation centrée et celle en intercuspidie maximale doit être fait dès l'étape pré-chirurgicale. On préfèrera l'occlusion en intercuspidie maximale à la relation centrée si la dimension verticale d'occlusion n'est pas perturbée et s'il subsiste un calage dento-dentaire lors de l'enregistrement des relations intermaxillaires. Dans le cas présenté, il n'y avait plus de calage prémolomolaire, l'occlusion en relation centrée était donc indiquée.

Ainsi, comme le montrent ce cas clinique et l'argumentation, la réhabilitation complète d'un maxillaire demande une collaboration étroite entre le chirurgien, le praticien prothésiste et le laboratoire de prothèse pour obtenir un résultat optimal tant au niveau fonctionnel qu'esthétique, sans oublier l'équipe infirmière qui s'assure du confort et de l'antalgie du patient pendant cette intervention longue.

Si la procédure d'implantation post-extractionnelle avec mise en charge immédiate allie les avantages propres à chacune des deux techniques qui la composent, les difficultés de chacune d'entre elles se surajoutent. Des conditions anatomiques, difficiles à prévoir avec précision sans planification, risquent parfois de compliquer l'acte chirurgical et de modifier le positionnement et l'axe des implants. C'est là la limite de cette procédure qui n'utilise pas de guide chirurgical issu de la planification informatique, mais qui laisse un degré de liberté au chirurgien. Aussi l'œil averti du praticien prothésiste est indispensable en salle d'intervention pour valider les orientations du chirurgien.

Malgré tout, cette technique est aujourd'hui fiable et reproductible pour des praticiens confirmés en implantologie. Cependant, des études prospectives à long terme en cours sont à finaliser afin de confirmer les résultats satisfaisants que l'on observe aujourd'hui.

\section{Remerciements}

Ce cas clinique a été réalisé par le professeur J.-L. Veyrune et le docteur J. Duroux. La prothèse a été élaborée par M. Vasconcelos (Laboratoire ARV) que nous remercions pour son implication dans I'Unité fonctionnelle d'implantologie orale du service d'odontologie du CHU de Clermont-Ferrand. Nous rendons hommage au Dr Y. Douillard pour tout ce qu'il a apporté à cette Unité fonctionnelle.

\section{Bibliographie}

1. Schulte $W$, et al. Animal experiments on the question of healing around the Tübingen immediate implant. Dtsch Zahnarztl Z. 1978 May;33(5):326-31.
2. Hâmmerle CHF, Chen ST, Wilson Jr TG Consensus statements and recommended clinical procedures regarding the placement of implants in extractions sockets. Int J Oral Maxillofac
Implants 2004;19(suppl):26-8.

3. Schropp, et al. Bone Healing following immediate versus delayed placement of titanium implants into extraction sockets : a prospective clinical study. 
Int J Oral Maxillofac

Implants 2003;18(2):189-99.

4. Shibly O, Kutkut A, Patel N, Albandar JM.

Immediate Implants with Immediate Loading vs Conventional Loading: 1-Year Randomized Clinical Trial.

Clin Implant Dent Relat Res 2010, Oct 26.

5. Prasad DK, Shetty M, Bansal N, Hegde C. Crestal bone preservation: A review of different approaches for successful implant therapy. Indian J Dent Res 2011 Mar-Apr;22(2):317-23.

6. Polizzi G, et al. Immediate and delayed implant placement into extraction sockets: a 5-year report. Clin Implant Dent Relat Res 2000;2(2):93-9.

7. Schwartz-Arad D, Chaushu G.

Placement of implants into fresh extraction sites: 4 to 7 years retrospective evaluation of 95 immediate implants.

J Periodontol 1997 Nov;68(11):1110-6.

8. Cohran DL, Morton D, Weber HP.

Consensus statements and recommended clinical procedures regarding loading protocols for endosseous dental implants. Int J Oral Maxillofac Implants 2004;19(suppl):109-13

9. Jayme SJ, de Oliveira RR, Muglia VA, Novaes AB Jr, Ribeiro RF.

The effects of different loading times on the bone response around dental implants: a histomorphometric study in dogs.
Int J Oral Maxillofac

Implants

2010 MayJun;25(3):473-81.

10. Blanco J, et al.

Soft tissue dimensions in

flapless immediate implants

with and without

immediate loading:

an experimental study

in the beagle dog.

Clin Oral Implants Res

2011, Apr 15.

11. Ravindran DM, Sudhakar U, Ramakrishnan T,

Ambalavanan $\mathrm{N}$

The efficacy of flapless

implant surgery on

soft-tissue profile

comparing immediate

loading implants to delayed

loading implants:

A comparative clinical study.

J Indian Soc Periodontol.

2010 Oct;14(4):245-51.

12. Erkapers $M$, et al.

Patient satisfaction

following dental implant

treatment with immediate

loading in the edentulous

atrophic maxilla.

Int J Oral Maxillofac

Implants.

2011 Mar-Apr;26(2):356-64.

13. Schwarz $S$, Gabbert $O$,

Hassel AJ, Schmitter M, Séché $C$, Rammelsberg $P$.

Early loading of implants

with fixed dental

prostheses in edentulous

mandibles: 4.5-year clinical

results from a prospective

study.

Clin Oral Implants Res

2010 Mar;21(3):284-9.

14. Malo $P$, de Araújo Nobre $M$, Lopes A, Moss SM,

Molina GJ.

A longitudinal study of the survival of All-on-4 implants in the mandible with up to 10 years of follow-up.

J Am Dent Assoc

2011 Mar; 142(3):310-20.
15. Degidi M, Nardi D,

Piattelli A.

Prospective study with a 2-year follow-up on immediate implant loading in the edentulous mandible with a definitive restoration using intra-oral welding. Clin Oral Implants Res 2010 Apr 1;21(4):379-85.

16. Pomares $C$.

A retrospective study of edentulous patients rehabilitated according to the 'all-on-four' or the 'all-on-six' immediate function concept using flapless computer-guided implant surgery. Eur J Oral Implantol 2010 Summer;3(2):155-63.

17. Chee W, Jivraj S. Efficiency of immediate loaded mandibular full-arch implant restaurations. Clin Implant Dent Relat Res 2003;(51):52-6.

18. Li W, Chow J, Hui E, Lee PK, Chow R.

Retrospective study on immediate functional loading of edentulous maxillas and mandibles with 690 implants, up to 71 months of follow-up. J Oral Maxillofac Surg 2009 Dec;67(12):2653-62.

19. De Bruyn $H$, Van de Velde $T$, Collaert B.

Immediate functional loading of TiOblast dental implants in full-arch edentulous mandibles: a 3-year prospective study. Clin Oral Implants Res 2008 Jul;19(7):717-23.

20. Eccellente $T$, Piombino $M$, Piattelli A, D'Alimonte $E$, Perrotti V, lezzi G. Immediate loading of dental implants in the edentulous maxilla. Quintessence Int 2011 Apr;42(4):281-9. 
21. Tealdo $\mathrm{T}$, et al.

Immediate versus delayed loading of dental implants in edentulous maxillae: a 36-month prospective study. Int J Prosthodont 2011 Jul-Aug;24(4):294-302.

22. Galli F, Capelli M, Zuffetti F, Testori T, Esposito M. Immediate non-occlusal vs. early loading of dental implants in partially edentulous patients: a multicentre randomized clinical trial. Peri-implant bone and soft-tissue levels. Clin Oral Implants Res 2008 Jun; 19(6):546-52.

23. Peñarrocha-Diago MA, Maestre-Ferrín L, Demarchi $\mathrm{CL}$, Peñarrocha-Oltra D, Peñarrocha-Diago $\mathrm{M}$. Immediate versus nonimmediate placement of implants for full-arch fixed restorations: a preliminary study. J Oral Maxillofac Surg 2011 Jan;69(1):154-9.

24. Fortin $T$, Champleboux G, Bianchi S, Buatois $\mathrm{H}$, Coudert JL. Precision of transfer of preoperative planning for oral implants based on cone-beam CT-scan images through a robotic drilling machine.

Clin Oral Implants Res 2002 Dec;13(6):651-6.

25. Fortin $\mathrm{T}$, Isidori $\mathrm{M}$, Bouchet $\mathrm{H}$. Placement of posterior maxillary implants in partially edentulous patients with severe bone deficiency using CAD/CAM guidance to avoid sinus grafting: a clinical report of procedure. Int J Oral Maxillofac
Implants

2009 Jan-Feb;24(1):96-102.

26. Amorfini $L$, Storelli $S$, Romeo $\mathrm{E}$.

Rehabilitation of a dentate mandible requiring a full arch rehabilitation. Immediate loading of a fixed complete denture on 8 implants placed with a bone-supported surgical computer-planned guide:

a case report.

J Oral Implantol

2011 Mar;37 Spec No:106-13.

27. Berdougo $M$, Fortin $T$, Blanchet $\mathrm{E}$, Isidori $\mathrm{M}$, Bosson JL.

Flapless implant surgery using an image-guided system. A 1- to 4-year retrospective multicenter comparative clinical study. Clin Implant Dent Relat Res 2010 Jun 1;12(2):142-52.

28. Abad-Gallegos $\mathrm{M}$, et al. Complications of guided surgery and immediate loading in oral implantology: a report of 12 cases.

Med Oral Patho

Oral Chir Bucal

2011 Mar 1;16(2):e220-4.

29. de Oliveira RR, Novaes $A B J r$ Taba M Jr, de Souza SL, Papalexiou V.

The effect of bone condensation and crestal preparation on the bone response to implants designed for immediate loading:

a histomorphometric study in dogs.

Int J Oral Maxillofac Implants 2007 Jan-Feb;22(1):63-71.

30. Chiapasco M, Gatti C, Gatti F. Immediate loading of dental implants placed in severely resorbed edentulous mandibles reconstructed with autogenous calvarial grafts. Clin Oral Implants Res 2007 Feb;18(1):13-20.

31. Browaeys $\mathrm{H}$, et al. A Retrospective Analysis of Early and Immediately Loaded Osseotite Implants in Cross-Arch Rehabilitations in Edentulous Maxillas and Mandibles Up to 7 Years. Clin Implant Dent Relat Res 2011 Jul 11.

32. Shibly O, Patel N, Albandar JM, Kutkut A. Bone regeneration around implants in periodontally compromised patients: a randomized clinical trial of the effect of immediate implant with immediate loading. J Periodontol 2010 Dec;81(12):1743-51.

33. Jo HY, Hobo PK, Hobo S. Freestanding and multiunit immediate loading of the expandable implant: an upto-40-month prospective survival study. J Prosthet Dent 2001 Feb;85(2):148-55.

34. Cooper LF, Rahman A, Moriarty J, Chaffee N, Sacco D.

Immediate mandibular rehabilitation with endosseous implants: simultaneous extraction, implant placement, and loading. Int J Oral Maxillofac Implants 2002 Jul-Aug;17(4):517-25.

35. Lorenzoni M, Pertl C, Zhang K, Wimmer G, Wegscheider WA. Immediate loading of single-tooth implants in the anterior maxilla. Preliminary results after one year. 
Clin Oral Implants Res 2003 Apr;14(2):180-7.

36. Norton MR.

A short-term clinical evaluation of immediately restored maxillary TiOblast single-tooth implants. Int J Oral Maxillofac Implants 2004 Mar-Apr;19(2):274-81.

37. Romanos GE.

Present status of immediate loading of oral implants. J Oral Implantol 2004;30(3):189-97.

38. Jaffin RA, Kumar A, Berman CL. Immediate loading of dental implants in the completely edentulous maxilla: a clinical report. Int J Oral Maxillofac Implants 2004 Sep-Oct;19(5):721-30.

39. Alves CC, Correia AR, Neves $M$. Immediate implants and immediate loading in periodontally compromised patients-a 3-year prospective clinical study. Int J Periodontics Restorative Dent 2010 Oct;30(5):447-55.

40. Crespi $R$, Capparè $P$, Gherlone E.

A 4-year evaluation of the peri-implant parameters of immediately loaded implants placed in fresh extraction sockets.
J Periodontol 2010 Nov;81(11):1629-34.

41. Duroux J, et al. Enregistrement des rapports intermaxillaires en salle blanche : cas cliniques. Implant 2011;17:173-89.

42. Canullo L, Bignozzi I, Cocchetto R, Cristalli MP, lannello $\mathrm{G}$. Immediate positioning of a definitive abutment versus repeated abutment replacements in post-extractive implants: 3-year follow-up of a randomised multicentre clinical trial.

Eur J Oral Implantol 2010;3(4):285-96.

\section{SUMMARY}

\section{Complete fixed prosthetic rehabilitation : simultaneous extractions, implants placement and loading}

Christophe DESCHAUMES, Julien DUROUX, Didier COMPAGNON, Cédric HUARD,

Emmanuel NICOLAS, Sylvain OPÉ, Manuel VASCONCELOS, Pascal AUROY, Jean-Luc VEYRUNE, Maurice MORENAS

\section{Keywords \\ - dental implants \\ - one-stage surgery \\ - immediate loading}

The concept of immediate loading and that of implantation in fresh sockets both knew a craze these last fifteen years and numerous clinical studies validated of these two protocols. Since around ten years, more and more teams associated this two concepts to develop the immediate implantation with immediate loading. Besides the speed of the treatment, the absence of temporary removable prosthesis and the fast return of the function of mastication, this protocol facilitates the bone and mucosa healing. The aim of this report is to present the interests and the constraints of this concept and to show a protocol for a complete rehabilitation of a jaw. 